\title{
The Effect of Exercise Duration on Mobile Phone Dependence among College Students: The Mediating Role of Self-Control
}

\author{
Weihang Huo* \\ Guangdong Peizheng College, Guangzhou 510830, Guangdong Province, China \\ *Corresponding author: Weihang Huo, 570848029@qq.com
}

\begin{abstract}
This research focused on the social concern of college students' participation in physical exercise and mobile phone dependence. The research model and hypotheses in this study were constructed based on the self-control theory and by reviewing previous research. This research distributed questionnaires to 207 college students from a university in Guangzhou, China to obtain relevant data. Mediation analysis was employed to test the four hypotheses drawn from empirical and conceptual research. The results of this research showed that first, the longer the exercise duration of college students, the higher the level of their self-control, thus exercise duration has a significant positive impact on self-control. Second, increasing the exercise duration of college students had no effect on their mobile phone dependence. Third, the higher the level of selfcontrol, the lower the dependence on mobile phones, thus signifying that self-control has significantly negative impact on mobile phone dependence. Fourth, self-control plays a mediating role in the effect of exercise duration on mobile phone dependence. In conclusion, the longer the exercise duration of college students, the higher the level of self-control, in which it is able to significantly reduce the dependence on mobile phones.
\end{abstract}

Keywords: College Student; Self-control; Mobile phone dependence; Physical exercise; Exercise duration

Publication date: August 2021; Online publication: August 30, 2021

\section{Introduction}

Up to December 2020, according to the $47^{\text {th }}$ Statistical Report on China's Internet Development ${ }^{[1]}$, the number of mobile internet users in China had reached 986 million, amounting to $99.7 \%$ of China's netizens accessing the internet with students about $21 \%$. The weekly online duration per capita was 26.2 hours. In China, there is an overreliance on mobile phones or mobile phone addiction among college students, which has become a major concern of the society. Mobile phone dependence results in the helplessness to control their own time. At times, they will even feel restless or angry when they do not use their mobile phones. The dependence on mobile phones has already affected college students' academic achievements in addition to their physical and mental health. According to the 2018 Physical Exercise Guidelines for Americans, 2nd edition, adults need to do two types of physical activity each week which include muscle strengthening and health-aerobic activities. A research that was conducted revealed that the excessive using of mobile phones causes many negative effects, such as reducing sleep quality, worsening of eyesight, and even damaging physical and mental health ${ }^{[2]}$. However, physical activities are able to alleviate the above problems as well as promote physical and mental health. In another research, Zhong and other researchers suggested that college students' participation in physical exercises do not only increase their self-control, but also decrease their mobile phone dependence ${ }^{[3]}$. 
However, all these studies only focused on the impact of physical activities on mobile phone dependence; they did not indicate the types of physical activities that influence college students' mobile phone dependence neither the amount of physical activities that is required for college students to reduce their mobile phone dependence. At the present moment, there is no relevant research on the effect of exercise duration on mobile phone dependence. In addition, studies have shown that better self-control can help college students to reduce or improve bad behaviors. For this reason, this study attempted to explore whether exercise duration has an effect on mobile phone dependence and self-control. Moreover, this study also tried to explore whether self-control has a mediating effect in the relationship between exercise duration and mobile phone dependence in hope to provide theoretical guidance for educational organizations and universities to form effective teaching strategies for college students.

\subsection{Mobile phone dependence and physical exercise}

College students often use their smartphones to communicate with their friends, obtain information through networking platforms, and also involve in entertainment activities, such as playing online games and shopping. These activities meet their demands and interests. However, college students have been spending more and more time on these activities via their mobile phones, resulting in poor academic performance and reduced sleeping time. The excessive use of mobile phones among some college students has led to psychological disorders and some college students even feel uncomfortable when they do not access information via their smartphones. Physical exercise is able to improve college students' dependence on mobile phones. In a research, it has been suggested that physical exercise is able to effectively reduce college students' dependence on mobile phones ${ }^{[4]}$. Another research also concluded the same ${ }^{[5]}$. However, neither of these two studies proved how physical exercise influence college students' mobile phone dependence or the mechanism of the effect of physical exercise on mobile phone dependence. As understood, physical exercise has three main dimensions, which are exercise duration, intensity, and types of physical exercises. Therefore, this study attempted to explore how exercise duration affects college students' mobile phone dependence.

\subsection{Physical exercise and self-control}

Physical exercise does not only enhance physical fitness, but also improve concentration. A research that was conducted have shown that strengthening physical exercise can improve college students' mental health ${ }^{[6]}$. It is believed that participating in various physical activities require good self-control. Zhang and other researchers showed through their research that exercising has positive effect on self-control and may even alleviate aggressive behaviors among college students ${ }^{[7]}$. In another research, it was shown that individuals with high self-control trait spend more time on physical exercise ${ }^{[8]}$. The researchers have supported the mediating role of physical exercise in affecting other factors.

\subsection{Self-control and bad habits}

Self-control is the key skill of how and when to restrain one's desires, impulses, behaviors, or emotions. In other words, individuals can prevent themselves from doing something they do not want to do or from feeling something they do not want to feel, especially when being tempted. Individuals who have successfully achieved their goals in the face of temptation had to face the conflict of self-control. Only by controlling oneself to avoid temptations can one achieve their goals ${ }^{[9]}$. In order to prevent college students from indulging in the temptation of mobile phones, they need to build on their self-control. In a research on college students' self-control and mobile phone dependence, it suggested a significant negative correlation between the various dimensions of college students' self-control and mobile phone dependence 
${ }^{[10]}$. In another research, Zhang and other researchers ${ }^{[11]}$ revealed that college students' self-control plays an intermediary role in mobile phone dependence and academic procrastination. In addition, through physical exercise, college students' self-control improved along with a better inhibitory or alleviating effect on other bad habits. Zhu and other researchers found that more physical exercise among college students improves their self-control and reduces or alleviates dependence on smoking, in which a higher level of self-control is able to decrease smoking dependence ${ }^{[12]}$.

\subsection{Hypotheses and proposed model}

The purpose of this study is to examine the degree to which exercise duration is related to mobile phone dependence. The following hypotheses and an initial hypothesized model were proposed in this study:

Hypothesis 1: Increasing college students' exercise duration will decrease their mobile phone dependence.

Hypothesis 2: Increasing college students' exercise duration will improve their self-control.

Hypothesis 3: A high level of self-control will reduce mobile phone dependence.

Hypothesis 4: The effect of exercise duration on mobile phone dependence is mediated by selfcontrol.

\section{Methods}

\subsection{Sample}

A total of 242 college students from a university in Guangdong, China participated in this study. The final sample had a total of 207 participants, in which their responses were used for the data analysis. Out of 207 participants, $143(69.1 \%)$ were female while 64 (30.9\%) were male. The mean age of the participants was 20.41 years old $(\mathrm{SD}=1.04)$, ranging from 19 to 22 years old.

\subsection{Data collection}

This study included three latent factors (exercise duration, self-control, and mobile phone dependence). A total of 16 survey items were selected for measurement. The Self-Control Scale ${ }^{[13]}$ was used to assess selfcontrol in this study. The scale comprised of seven items (e.g., "When I do not want to exercise, I can still make myself do it"; "I will ignore the short-term benefits of sports") including two reverse-items (e.g., "I often want to stop doing sports"; "I do not think about the long-term benefits of sports"). Response scales of self-control were recorded using the 7-point Likert-type scale ( $1=$ Strongly disagree to 7= Strongly agree). Higher scores indicated a greater degree of self-control. In the first reliability test, Cronbach's $\alpha$ for selfcontrol was 0.61 but after adjustment with the deletion of one reverse-item, Cronbach's $\alpha$ was 0.72 ; the reliability of the 6 items was higher than 0.7 , indicating that the reliability of the Self-Control Scale in this study is accepted. Higher scores indicated a greater level of self-control.

The Mobile Phone Dependence Scale ${ }^{[14]}$ was adopted to measure mobile phone dependence. The scale comprised of 8 items (e.g., "I often think about my mobile phone when I am not using it"; "I have been unable to reduce my mobile phone usage"; "The thought of being without my mobile phone makes me feel distressed"; "I often use my mobile phone for no particular reason"). Response scales of mobile phone dependence were recorded using the 7-point Likert-type scale ( $1=$ Strongly disagree to $7=$ Strongly agree). Higher scores indicated a greater level of mobile phone dependence. Cronbach's $\alpha$ for mobile phone dependence was 0.75 , in which the reliability of the 8 scales was higher than 0.7 , indicating that the reliability of the Mobile Phone Dependence Scale is accepted.

The exercise duration factor was measured by using one item that comprised of five options (e.g., "below 10 minutes"; "between 20 and 40 minutes"; "over 60 minutes"). 


\subsection{Data analysis}

Descriptive analyses and Pearson correlations were conducted using Statistical Package for the Social Sciences (SPSS) version 22.0 and the mediation model was tested using SPSS macro process, which was developed by Hayes ${ }^{[15]}$. The common method variance was also estimated, in which the Harman single factor test was adopted to test the common method variance. The results revealed that the variables were without rotation, five factors with roots of each trait greater than 1 , and the interpretation percentage of the first common factor was $25.2 \%$, which is less than the critical standard of $40 \%$. It has been considered that there was no serious common method bias.

\section{Result and discussion}

Descriptive statistics and correlation estimation were conducted. The analysis results are shown in Table 1. The results revealed that the college students' exercise duration was positively related to their self-control, and their self-control was negatively related to their mobile phone dependence. However, their exercise duration was not related to their mobile phone dependence $(p=0.07>0.05)$. This means that increasing or decreasing the exercise duration of college students has no impact on their mobile phone dependence. Hence, Hypothesis 1 is not supported.

Thereafter, the proposed model was examined by using the Model 4 of SPSS macro process. The proposed model comprised of three parts: Model 1, Model 2, and the conditional indirect effect analysis of Model 2. The results of the data analysis are presented in Table 2. The results of Model 1 revealed that exercise duration had significantly positive effect on self-control $\left(F=29.86, R^{2}=0.13, \beta=0.37, p<0.01\right)$, thus supporting Hypothesis 2 . The results also revealed a significant conditional indirect effect on mobile phone dependence $\left(\mathrm{F}=7.16, R^{2}=0.07, \beta=-0.24, p<0.01\right)$, thus supporting Hypothesis 3.

Finally, the mediating role of the self-control factor between exercise duration and mobile phone dependence (Model 2) is tested. The results of the conditional process analysis revealed that the indirect mediation effect was statistically significant (95\% confidence interval of -0.1582 to -0.0397 , not containing zero) in terms of the mediation effect of the self-control factor between exercise duration and mobile phone dependence, thus supporting Hypothesis 4. However, the direct mediation effect was not statistically significant ( $95 \%$ confidence interval of -0.0702 to 0.1905 , containing zero) in terms of exercise duration in directly predicting mobile phone dependence. Self-control had a mediating effect through exercise duration on mobile phone dependence. In general, based on the results of mediation estimation, self-control played a partial mediating role between exercise duration and mobile phone dependence.

Table 1. Means and correlations between variables

\begin{tabular}{lcccc}
\hline \multicolumn{1}{c}{ Variables } & Mean & SD & 1 & 2 \\
\hline Exercise duration & 3.47 & 1.07 & - & - \\
Self-control & 4.43 & 1.11 & $0.36^{* *}$ & - \\
Mobile phone dependence & 3.77 & 0.98 & -0.03 & $-0.25^{* *}$ \\
\hline
\end{tabular}

Note. $* \mathrm{p}<0.05$. **p $<0.01$. 
Table 2. Conditional process analysis

\begin{tabular}{llcccccc}
\hline Outcome & Predictors & $\beta$ & $s e$ & $t$ & $p$ & LLCI & ULCI \\
\hline Model 1 & & & & & 0.00 & & \\
Self-control & Exercise duration & 0.37 & 0.07 & 5.46 & 0.00 & 0.2354 & 0.5012 \\
Model 2 & & & & & & \\
Mobile phone dependence & Self-control & -0.24 & 0.06 & -3.76 & 0.00 & -0.3668 & -0.1144 \\
& Exercise duration & 0.07 & 0.07 & 0.91 & 0.36 & -0.0702 & 0.1905 \\
\hline Conditional indirect effect & & $\beta$ & Boot SE & Boot LLCI & Boot ULCI \\
\hline Mediation & -0.09 & 0.03 & -0.1582 & -0.0397 \\
\hline Conditional direct effect & & & & & \\
\hline Mediation & 0.06 & 0.07 & -0.0702 & 0.1905 \\
\hline
\end{tabular}

Note. Bootstrap sample size: 5000; LL: low limit; CI: confidence interval; UL: upper limit.

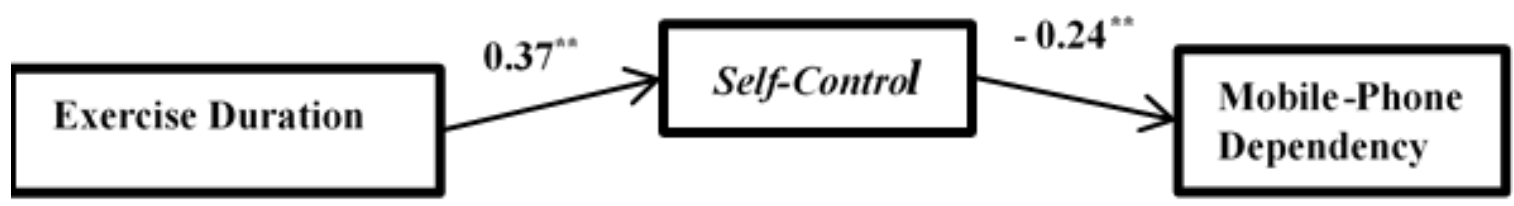

Figure 1. Effect of exercise duration on mobile phone dependence

\section{Conclusion}

This study conducted an extended research based on previous research on the effect of exercise on selfcontrol and bad habits. The relationship among exercise duration, self-control, and mobile phone dependence is clarified. The results of this study suggested that increasing the exercise duration of college students is able to effectively improve their self-control, thus decreasing the dependence on mobile phones. However, the results also revealed that exercise duration does not directly affect college students' mobile phone dependence. First, the result of increasing exercise duration improves self-control is similar to Liu's research ${ }^{[6]}$, whereby physical exercise improves self-control. However, Liu's research did not point out which specific features of physical exercise can improve self-control. The result of this study revealed that, in which increasing the exercise duration improves self-control. Second, the result whereby higher level of self-control reduces mobile phone dependence is similar to the research conducted by Zhang, et al. and Zhu ${ }^{[4,11]}$. All studies have suggested that individuals with better self-control are able to reduce or change bad habits, such as smoking and mobile phone dependence, as well as restrain short-term temptations.

The results of this study allow further understanding of the factors of mobile phone dependence among college students, as well as how to reduce their dependence on mobile phones. The results also provide a practical guiding significance for relevant educational organizations and committees to implement appropriate strategies and policies to reduce students' mobile phone dependence. Based on the results of this research, educational organizations such as schools, colleges, universities should arrange ample time for students to participate in physical exercise every day. Students who participate in physical exercise should not only take into consideration of the intensity of the exercise, but also the duration.

The results of this study may provide further understanding on the effect of physical exercise on both self-control and mobile phone dependence, as well as implications for bad habits due to poor self-control.

\section{Disclosure statement}

The author declares that there is no conflict of interest. 


\section{References}

[1] China Internet Network Information Center (CNNIC), 2021, The $47^{\text {th }}$ Statistical Report on China's Internet Development. CNNIC (updated February 2021).

[2] Fu X, Lin Y, 2019, Studying the Negative Effects of Mobile Phone on Users' Health: A Large-Scale Survey of College Student. Documentation, Information \& Knowledge, (02): 120-9.

[3] Zhong W, Wang Y, Zhang G, 2020, The Impact of Physical exercise on College Students' Mobile Phone Dependence: The Mediating Role of Self-Control. International Journal of Mental Health and Addiction, : 1-16.

[4] Zhu G, 2017, Study on the Intervention of Physical Exercise on College Students' Tendency of Mobile Phone Addiction. Zhejiang Sport Science, 39(5): 90-7.

[5] Bu Y, 2014, Effect of Physical Exercise on College Students' Mobile Phone Addiction. Modern Preventive Medicine, 41(7): 1242-4.

[6] Liu H, 2012, Strengthen Physical Exercise and Promote Students' Mental Health. Journal of Changchun Education Institute, (22): 108-9.

[7] Zhang Z, Zhu F, Cai X, 2018, Mediation Role of Self-Control in the Association between Physical Exercise and Aggressive Behavior among College Freshmen. Chinese Journal of School Health, 39(07): 1037-40.

[8] Schöndube A, Bertrams A, Sudeck G, et al., 2017, Self-Control Strength and Physical Exercise: An Ecological Momentary Assessment Study. Psychology of Sport and Exercise, 29: 19-26.

[9] Myrseth K, Fishbach A, 2010, Self-Control: A Function of Knowing When and How to Exercise Restraint. Current Directions in Psychological Science, 18(4): 247-52.

[10] Li Y, Jin L, Zhang S, 2016, Research on the Relationship between Self-Control Ability and Mobile Phone Dependence among University Students. Chinese Journal of Health Education, 32(9): 775-8.

[11] Zhang C, Zhai L, Wang C, 2017, Mediating Effect of Self-Control on the Relationship between Mobile Phone Dependence and Academic Procrastination in College Students. China Journal of Health Psychology, 25(1): 145-8.

[12] Zhu F, Zhou C, Wang B, 2014, Physical Exercise Decreases the Smoking Dependence of College Students: Mediation Effect of Self-Control. Sports \& Science, (6): 109-13.

[13] Giner-Sorolla R, 2001, Guilty Pleasures and Grim Necessities: Affective Attitudes in Dilemmas of Self-Control. Journal of Personality and Social Psychology, 80(2): 206.

[14] Walsh P, White M, et al., 2008, Dialing and Driving: Factors Influencing Intentions to Use a Mobile Phone While Driving. Accident Analysis \& Prevention, 40(6): 1893-900.

[15] Hayes AF, 2013, Introduction to Mediation, Moderation, and Conditional Process Analysis: A Regression-Based Approach, The Guilford Press, New York, 76-86.

[16] Guay F, Vallerand J, Blanchard C, 2000, On the Assessment of Situational Intrinsic and Extrinsic Motivation: The Situational Motivation Scale. Motivation and Emotion, (24): 175-212. 